\title{
Intelligent PID Controller of Flexible Link Manipulator with Payload
}

\author{
A. Jamali \\ Faculty of Engineering \\ Universti Malaysia Sarawak \\ Kota Samarahan Sarawak, Malaysia \\ jannisa@unimas.my
}

\author{
Mat Darus I. Z. \\ Faculty of Mechanical Engineering \\ Universiti Teknologi Malaysia \\ Skudai, Johor Bahru, Malaysia \\ intan@utm.my
}

\author{
M. O Tokhi \\ School of Engineering \\ London South Bank University, \\ London \\ tokhim@1sbu.ac.uk
}

\begin{abstract}
This paper presents the experimental study of intelligent PID controller with the present of payload. The controllers were constructed to optimally track the desired hub angle and vibration suppression of DLFRM. The hub angle and end-point vibration models were identified based on NNARX structure. The results of all developed controllers were analyzed in terms of trajectory tracking and vibration suppression of DLFRM subjected to disturbance. The simulation studies showed that the intelligent PID controllers have provided good performance. Further investigation via experimental studies was carried out. The results revealed that the intelligent PID control structure able to show similar performance up to $20 \mathrm{~g}$ of payload hold by the system. Once the payload increased more than $20 \mathrm{~g}$, the performance of the controller degrades. Thus, it can be concluded that, the controllers can be applied in real application, provided the tuning process were carried out with the existence of the maximum payload which will be subjected in the system. The $20 \mathrm{~g}$ payload value can act as uncertainty for the controller performance.
\end{abstract}

Keywords-flexible manipulator, payload, robotic manipulator, intelligent controller, PSO.

\section{INTRODUCTION}

The flexible plate has been utilized in various industry applications such as aircraft body, automotive structure, robotic arm, electronic board design, bridge decks, and conveyor system [1]. Many researchers have contributed in the study of flexible plate such as in modeling [2-4] and control strategies [5-7].

Recently, the spectrum of applications of flexible plate in robotic arm has become wider and greater. It was used on the land, underwater and outer space. The broader scope of usage shows that the flexible manipulator is practical for real application. It becomes favorable alternative as it is recognized to be exceptionally productive. In opposite to the inflexible structure, it offers lighter in weight, consume less power, require smaller actuators, transportable, higher maneuverability, more secure operation and lower natural affect. However, the structure produces undesirable vibration from the flexible structure which give a lot of effort in controlling the structure. The challenge in control task become intensifies when the flexible multi-link is considered. Aside from the vibration exist in the system, several other factors such as coupling between both links have to be carefully measured. Due to that, the control scheme must be effective and robust.

Flexible Manipulator captures significant interest among researchers in order to accommodate the current industry's needs. The existing researches focused on enhancing the control techniques so that it can comply with all the contradictory needs. In the industrial environment, proportional integral derivative (PID) controller is still a promising choice particularly for multi input multi-output (MIMO) frames. They are frequently showed satisfactory accomplishment despite their simple design. A group of researchers has proposed an easy tuning method. There are three classes of decentralized PID manage method that is detuning of the parameter, in search of on essential gains and acquired controllers through analytic, numerical or graphics methods via taking into accounts the interaction consequences [8]. Meanwhile, additional detail category has been further explained in [9] that is detuning methods, sequential loop closing methods, iterative or trial-and-error methods, simultaneous solving equation or simultaneous optimization methods and unbiased methods.

Several of PID control scheme have been implemented to double link flexible robotic manipulator (DLFRM) system. The work in [10-11] have proposed the PI-PID controller for DLFRM through manual tuning. Then, the overall performance has been elevated by adding ILC which have been verified in the simulation. The linear matrix inequalities (LMI) based PID control of a nonlinear Double link flexible robotic manipulator (DLFRM) incorporating payload have been investigated which is categorized under simultaneous equation optimization method [12]. In [13] PID controller by incorporating bounding parameters of interconnection terms in LMI formulation for an n-link robotic manipulator system was proposed. Another control strategy for DLFRM utilized Neural Network (NN) to approximate the ZN-PID for every link of DLFRM in [14] which can be classified beneath Independent method. Besides, PID tuned by P-type ILA is proposed for DLFRM [15]. The outcome showed a convincing result. Finally, PID tuned by PSO and ABC are offered. The results showed that both the intelligent controllers successfully control the DLFRM but PSO superseded ABC [16].

Looking at the wider study dimension of DLFRM, the research on a single link flexible manipulator (SLFM) can be explored. There are quite a number of researches have been conducted for SLFM. Alam, et al. applied hybrid PD-PD/ILA tune by multi-objective Genetic Algorithm optimization for SLFM [17]. Tijani, carried out a multi-objective optimization the use of Differential Evolution (MODE) for PID controller of SLFM [18]. Another researcher has proposed an expanded Bacterial Foraging Algorithms (BFA) to fine-tune the PID controller of SLFM [19]. Bee Algorithm have been successful to optimize the hierarchical PID parameter of 